\title{
Reliability of Passive Hip Extension Mechanical Parameters in Healthy Subjects
}

\author{
Anabèle Brière, Denis Gravel and Sylvie Nadeau* \\ École de Réadaptation, Faculté de Médecine, Université de Montréal and Centre de Recherche Interdisciplinaire en \\ Réadaptation du Montréal Métropolitain (CRIR), Institut de Réadaptation Gingras-Lindsay-de-Montréal, QC, H3C 3J7, \\ Canada
}

\begin{abstract}
Objective: The objective was to estimate the reliability of three stiffness parameters (moment, angle and stiffness coefficient) of the hip extension movement, for three knee positions

Design: Twenty healthy subjects were assessed on two days separated by a one-week interval. A modified Biodex dynamometer was used to move the right lower limb passively in a side-lying position and to record the hip angle and moment during the movement. Three trials with the knee positioned at $0^{\circ}, 45^{\circ}$ and $90^{\circ}$ of flexion were performed each day. Passive stiffness was quantified by three parameters derived from the moment-angle curve of each subject. The generalizability theory was used to determine the reliability coefficients and standard errors of measurement (SEM) computed for a design comprising five trials in one day.

Results: Overall, the reliability coefficients were moderate to good with values ranging from 0.58 to 0.83 at the three knee positions. The highest SEM values were $4.15 \mathrm{Nm}, 8.4^{\circ}$ and $0.15 \mathrm{Nm} /{ }^{\circ}$ for the moment, angle and stiffness coefficient parameters, respectively. The main source of error variance was related to the subject-day interaction factor and a systematic day effect was also noted.

Conclusions: This study constitutes a first step towards objective evaluation of hip extension joint stiffness. Future studies will be needed to better control the alignment of the hip to reduce the systematic day error on the stiffness parameters.
\end{abstract}

Keywords: Joint stiffness, hip, reliability, Biodex system, healthy subjects, generalizability theory, stiffness parameters.

\section{INTRODUCTION}

Abnormal joint stiffness and muscular contractures are frequently observed in patients with motor impairments [1$3]$. It has been shown that these deficiencies decrease an individual's ability to perform functional activities such as walking [1,2]. Increased joint stiffness can also be present in healthy subjects as well as in athletes. For these individuals, joint stiffness can affect their sport performance and may increase the risk of muscular-skeletal injuries [4]. In this context, it is imperative to study the reliability of passive stiffness measures in healthy subjects to appreciate the differences between normal subjects and patients, to evaluate the effects of stretching interventions, to demonstrate factors that correlate with passive stiffness as well as to establish the real contribution of passive moment during functional tasks such as walking. Reliable measurements will help to achieve these experimental and clinical goals.

There are many anatomical structures that contribute to passive joint stiffness. In a study investigating the relative importance of various tissues to passive stiffness of the cat's

*Address correspondence to these authors at the École de Réadaptation, Faculté de Médecine, Université de Montréal and Centre de Recherche Interdisciplinaire en Réadaptation du Montréal Métropolitain (CRIR), Institut de Réadaptation Gingras-Lindsay-de-Montréal, QC, H3C 3J7, Canada; Tel: (514) 343-2253; Fax: (514) 343-2105;

E-mail: sylvie.nadeau@umontreal.ca wrist, Johns and Wright [5] reported that the contributions of the skin, tendon, capsule and muscle to the total stiffness measured in the mid-range of motion were $2 \%, 10 \%, 47 \%$ and $41 \%$, respectively. Obviously, the importance of each tissue cannot be determined in human joints, but the muscles and their surrounding connective tissues (endomysium, perimysium and epimysium) appear to be significant factors to consider [6]. Indirect evidence of the importance of these structures is provided by the influence of the angular positions of adjacent joints on stiffness measured at a joint. At the hip joint, it was observed that the stiffness associated with the flexion movement is increased by the extension of the knee $[7,8]$ and this was attributed to the lengthening of the hamstring muscles. A similar phenomenon was demonstrated at the ankle, where the stiffness associated with the dorsiflexion movement is increased by the knee extension position [9]. In this case, it was inferred that the lengthening of the gastrocnemius muscles caused this increase in stiffness.

The present study concerns the evaluation of hip extension passive stiffness. This is particularly relevant because of the limited range of motion of hip extension in humans. Indeed, the entire available range of hip extension is used during gait where full extension is reached at the end of the push-off phase. At this point, the contribution of the passive moment to the total gait moment was estimated to be near $10 \%$ [7] to $30-50 \%$ [8]. Thus, the passive stiffness could be a positive factor in gait because it will reduce the active con- 
tribution of muscle. On the other hand, if stiffness is too high, hip extension will be decreased and gait performance will be modified [3, 10,11]. Because one aspect of our scientific program is to determine the positive and negative role of passive hip stiffness on gait performance, the present reliability study was planned to find the error associated with experimental stiffness measurements.

Until now, only three studies have investigated the passive stiffness at the hip during the extension movement [7, 8, 12]. These studies on healthy subjects demonstrated that the passive moment increases non-linearly with the augmentation of hip extension. In all of these studies, the hip extension moment was increased by a flexed knee position and this result was explained by the presence of the biarticular rectus femoris muscle.

Among these three studies, only one evaluated the consistency of their method [7]. Vrahas et al. [7] conducted repeated measurements on five subjects (two subjects were tested on three different days, and three additional subjects were tested on two different days) and they found a typical variability from day to day of 2 N.m. However, for some angles, the average maximum variability was 4.4 N.m.

The previous studies measured hip passive stiffness using only the moment parameter. However, passive stiffness can also be evaluated by two other stiffness parameters derived from the moment-angle curve $[13,14]$. The first is the angle measured for a specific moment [13]; this parameter may be useful if comparisons are to be made with goniometric angles measured by clinicians. The second parameter is the stiffness coefficient (slope of the moment-angle curve) calculated at a specific angle [13]. This parameter is often reported in the literature because it integrates the moment and angle values. However, a common reference point (moment or angle) has to be determined in order to compare subjects on their level of stiffness or to compare stiffness measures before and after a stretching program. For example, a subject will be considered stiffer than another if the passive moment recorded at a similar or common angle is higher, if the angle measured at a common moment is smaller, or if the stiffness coefficient is higher for a common angle [13, 14].

To our knowledge, no study has been done on the reliability of experimental hip stiffness measurements in healthy subjects including a larger number of subjects and carried out with an appropriate reliability methodology. The purpose of this study was to estimate the reliability of three passive stiffness parameters (Moment, Angle and Stiffness Coefficient) for the hip extension movement in healthy subjects for three knee positions. A test-retest design with multiple trials was used to establish the reliability associated with Trial and Day factors. The generalizability theory [15] served as the framework to determine the sources of variance (G-study) as well as the level of reliability and errors expected for a particular design (D-study). Because the setup will not be changed between trials but will between days, it is expected that the inter-trial reliability will be lower than the betweenday reliability. Considering the data reported by Vrahas et al. [7], the between-day reliability will be acceptable (reliability coefficients $>0.75$ ) with a standard error of measurement (SEM) lower than $5 \mathrm{Nm}$. The knee position will not affect the reliability level because we believe that the between- person and error variances (the component of the reliability coefficient) will change similarly across knee positions.

\section{MATERIALS AND METHODOLOGY}

\section{Subjects}

A convenience sample of 20 healthy subjects (12 women and $8 \mathrm{men}$ ), aged between 20 and 63, participated in the present study (Table 1). All of the participants presented normal right lower limb range of motion according to a global evaluation and were free of any back or lower extremity problems. They were employees of the Montreal Rehabilitation Institute and students of the University of Montreal. Written informed consent was obtained before participation in the study as approved by the ethics committee of the Centre de recherche interdisciplinaire en réadaptation du Montréal métropolitain.

Table 1. Subject Characteristics (Means, 1 Standard Deviations and Ranges)

\begin{tabular}{|c|c|c|c|}
\hline Characteristics & Mean & Standard Deviation & Range \\
\hline \hline Age (years) & 38.2 & 14.4 & $20-63$ \\
\hline Body Mass $(\mathrm{kg})$ & 67.0 & 14.5 & $52-104$ \\
\hline Height $(\mathrm{cm})$ & 159.2 & 37.8 & $148.5-192.5$ \\
\hline
\end{tabular}

\section{Study Design}

A test-retest design was used to assess the reliability of the stiffness parameters. Each subject was assessed at the same time of day on two days, separated by a one-week interval. Hip stiffness was evaluated at three knee positions and the testing sequence of these positions was randomized across the subjects. For each subject, this sequence was the same both days. On the first day, a clinical exam was done to verify that the lower limb had no obvious deformation or orthopaedic problems not reported by the subject and to gather information on their physical characteristics (anthropometrical measures and inferior limb range of motions). In addition, measures related to the position of the subject and apparatus (alignment of subject and dynamometric component adjustments) were taken on the first assessment and were used to ensure a similar positioning of the subjects at the second evaluation.

\section{Procedures}

\section{Experimental Apparatus}

The experimental apparatus included a Biodex dynamometer used to move the subject's hip through the maximum permissible range of flexion and extension at a constant velocity $(15 \%)$ while simultaneously measuring the passive moment as well as the position of the evaluated hip. The dynamometer was connected with two pulleys and steel cables to a suspension system fixed on a rigid metal frame. The suspension system was used to support the lower limb during its displacements, which occurred in a horizontal plane with the subject placed in a lateral decubitus position (Fig. 1A). This position eliminated the influence of gravity 
on the moment recorded at the hip. Supports were provided at the thigh and at the ankle to easily change the knee position and to facilitate muscle relaxation.

(A)

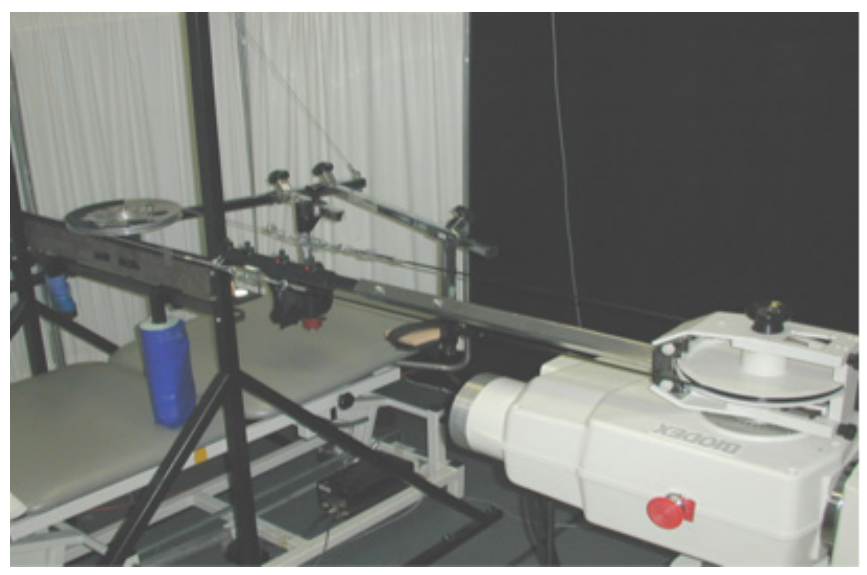

(B)

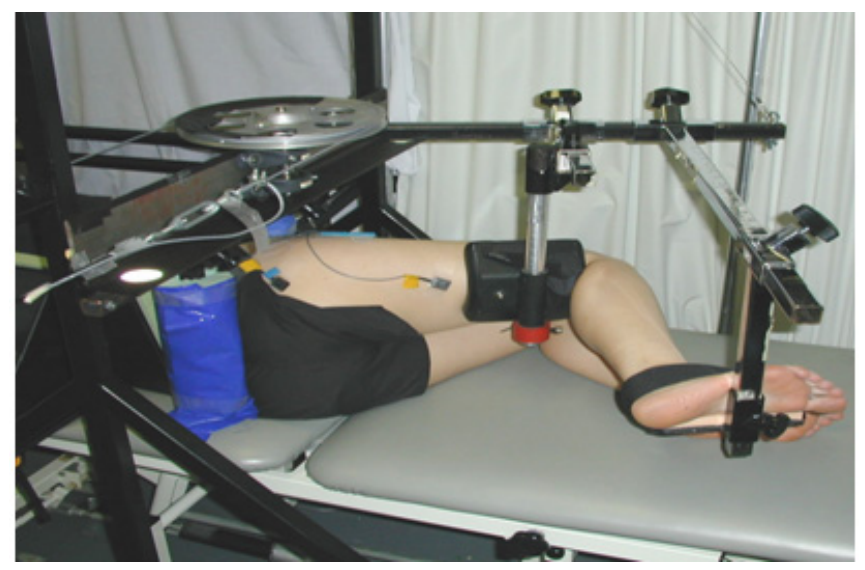

Fig. (1). Experimental hip stiffness measurements. (A) Experimental apparatus. (B) Subject positioning.

In addition, electromyographic (EMG) activities of the hip muscles were recorded by active bipolar electrodes [Model DE-2.3, DelSys Inc. Wellesley, MA] and amplified by a Nihon Kohden module [Model AM-601G]. The amplifiers had a noise background of less than $6 \mu \mathrm{V}$ and the bandwidth was set at 1-500 Hz. Biodex dynamometric and EMG signals were sampled at $1200 \mathrm{~Hz}$.

\section{Subject Preparation and Positioning}

After an appropriate preparation of the skin, EMG electrodes were placed longitudinally on the following muscles: rectus femoris, tensor fasciae latae, semimembranosus, biceps femoris, gluteus maximus and lumbar spinae erector. With the subject in a left lateral decubitus position, the flexion/extension axis of the right hip was aligned with the rotation axis of the pulley driving the support system. Then, the right lower limb was fixed into the supports and the first knee position was adjusted. The subject's left limb (nontested) was strapped to the table with the hip flexed at $45^{\circ}$ and the knee at $90^{\circ}$. The pelvis was stabilized by two cushioned supports clamped firmly against the lumbosacral region and the anterior superior iliac spines to limit its movements as much as possible during the hip motion (Fig. 1B).

\section{Testing Procedures}

A first data acquisition was taken at rest with the hip in a neutral position $\left(0^{\circ}\right)$, that is, with the trunk and thigh aligned in the same frontal plane. This acquisition was done to make sure that every subject would have the same hip position from one day to the other and it gave the reference angle to construct the moment-angle curves. A second data acquisition of $30 \mathrm{~s}$ was done at rest in a $30^{\circ}$ hip flexion position to record the basic EMG activity level of the six evaluated muscles. This recording was later used in the data analysis to verify the level of muscle activity during the passive movements.

Afterwards, measures of hip passive stiffness were taken for the three knee positions. These were with the knee in complete extension $\left(\mathrm{K}^{\circ}\right)$, in a $45^{\circ}$ flexion position $\left(\mathrm{K} 45^{\circ}\right)$ and in a $90^{\circ}$ flexion position $\left(\mathrm{K} 90^{\circ}\right)$. For each of these positions, the evaluator determined the maximum range of hip motion tolerated by the subject and the limits in flexion and extension were fixed on the Biodex dynamometer. Three 60 $\mathrm{s}$ trials separated by a 1-min rest period were recorded for each knee position. During each of these trials, two to three passive oscillation phases of flexion and extension were completed and each change of direction between these phases lasted $1 \mathrm{~s}$. After completion of three trials for each of the three knee positions, a fourth trial was recorded without the subject in the suspension system within the same range of motion. This recording was taken to correct the measures of stiffness for the moment created by the suspension system.

\section{Data Processing}

\section{General Data Processing}

All the data (EMG signals, moment, velocity and position values) were processed with software developed at our research center to keep only the one recorded during the extension phases. The EMG signals were full-wave rectified and then filtered by a digital low-pass Butterworth filter with a cut-off frequency of $3 \mathrm{~Hz}$. Magnitude of the EMG signal was compared to that of the resting trial. Recordings having values greater than twice the EMG resting level were discarded from the analysis. The moment signal was filtered at $1 \mathrm{~Hz}$ to eliminate the mechanical vibrations present at the beginning of each phase. The next step was to re-sample all signals in order to have values at each degree of movement. Then, at each angle, the moment created by the suspension system was subtracted from the recorded total moment, to obtain the moment generated only by the passive structures surrounding the hip. Finally, the mean of two extension phases was calculated to obtain the moment-angle curve for each trial. The stiffness coefficient was calculated by subtracting the moment value found at a given angle from the moment found at the preceding angle. Therefore, the units of the stiffness coefficient were defined as N.m/degree.

\section{Data Selection}

Since every subject had a specific moment-angle curve with a different maximal angle of extension and maximal moment value, it was important to define data points on the moment-angle curve that were common to all subjects. For example, to compute the moment as the relevant variable, an 
angle common to all subjects must be identified. To illustrate this approach, Fig. (2) presents an example with the moment-angle curves of two subjects recorded during the hip extension movement with the knee in a $90^{\circ}$ flexion position $\left(\mathrm{K} 90^{\circ}\right)$. The vertical line at $1^{\circ}$ indicates the common extension angle at which the moment value for each subject was taken. It is important to note that the $1^{\circ}$ angle was the maximal one found on the curve of subject \#1 which determined, in this example, the common angle for the two subjects. Extending this example to the sample of this study, the common angle was the lowest one found among all subjects. The same reasoning holds for the stiffness coefficient at a common angle as well as for the angle at a common moment. Obviously, for each subject and for the same knee position, the common moment or the common angle was the same across days because a test-retest design is used in the present study. However, these common angles and moments were not the same for the three knee positions because reliabilities were calculated independently for each knee position.

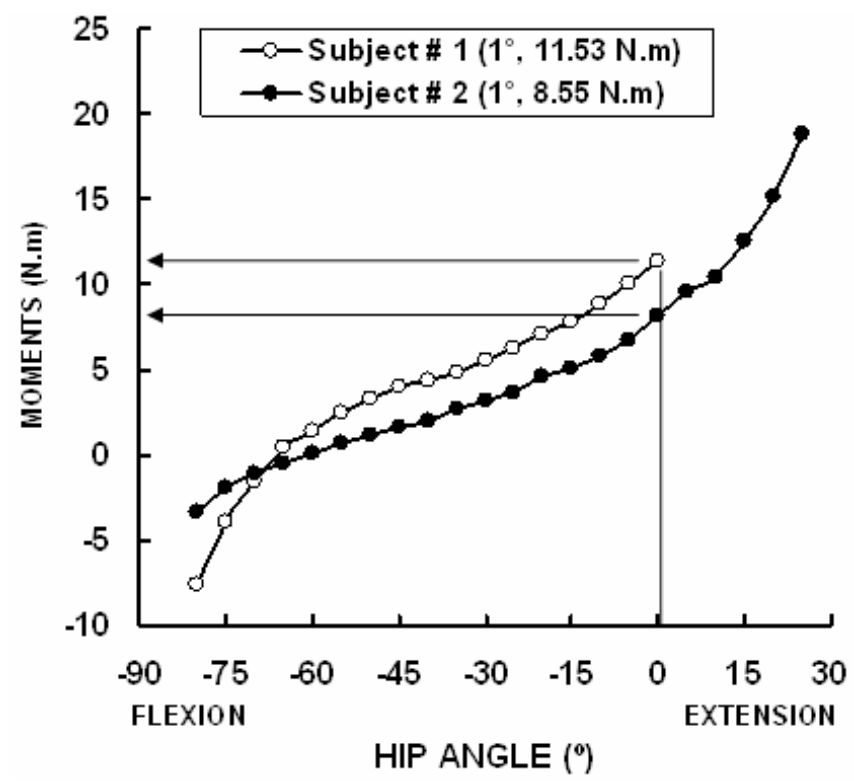

Fig. (2). Moment-angle curves of two subjects (subject \#1 and subject \#2) recorded during the hip extension movement with the knee in a $90^{\circ}$ flexion position $\left(\mathrm{K} 90^{\circ}\right)$. The vertical line at $1^{\circ}$ indicates the common extension angle at which the moment value for each subject was taken.

\section{Statistical Analysis}

The reliability for the stiffness measures was evaluated by the generalizability theory for a crossed design where each subject was exposed to the same repeated measurement conditions [15]. In this theory, multiple sources of errors, either systematic or random, can be considered. Random errors are due to pure chance happenings whereas systematic errors are present across subjects. In the generalizability theory, the subject is the object of measurement and the factors, called facets, are the conditions of the measure.

The generalizability theory is based on the analysis of variance. It is divided into two steps: the first is the generalizability study (G-study) and the second, the decision study (D-study). The G-study aims at identifying the magnitude of the variances attributed to the Subject $\left(\sigma^{2}\right)$, to the systematic errors related to the Day $\left(\sigma_{D}^{2}\right)$ and Trial $\left(\sigma_{T}^{2}\right)$ facets as well as to the random errors associated with the interactions between variance components. These interactions include the Day-Trial $\left(\sigma_{D T}^{2}\right)$, Subject-Day $\left(\sigma_{S D}^{2}\right)$, Subject-Trial $\left(\sigma_{S T}^{2}\right)$ and the residual error defined by the interaction between all sources of error, i.e. Subject-Day-Trial $\left(\sigma_{S D T}^{2}\right)$. All these variances can be expressed as percentages of the total variance obtained by the summation of the above variances.

The D-study uses the information of the G-study to determine the reliability of a particular protocol. In the present study, the hypothetical protocol retained is one where five trials are done on one day. Two reliability coefficients will be reported, the dependability indices and the generalizability coefficients. The reliability coefficient, defined by Brennan and Kane [16] as a dependability index $(\Phi)$, is a ratio of the inter-subject variance $\left(\sigma_{S}^{2}\right)$ to the total variance that sums the inter-subject variance $\left(\sigma_{S}^{2}\right)$ and the absolute error variance $\left(\sigma_{A B S}^{2}\right)$. This last term is composed of the six error variances (systematic and random) described in the preceding paragraph. The dependability index for a D-study using the mean of five trials in one day is given by:

$$
\begin{aligned}
& \Phi=\frac{\sigma_{\mathrm{S}}^{2}}{\sigma_{\mathrm{S}}^{2}+\sigma_{\mathrm{ABS}}^{2}} \text { where } \\
& \sigma_{A B S}^{2}=\left(\sigma_{D}^{2}\right)+\left(\sigma_{T}^{2}\right) / 5+\left(\sigma_{D T}^{2}\right) / 5+\left(\sigma_{S D}^{2}\right)+\left(\sigma_{S T}^{2}\right) / 5+\left(\sigma_{S D T}^{2}\right) / 5
\end{aligned}
$$

Since the systematic variance is not relevant in some forms of research (c.f.: correlational study), the generalizability coefficient will also be presented. This coefficient considers only the random error variances associated with the subject factor $\left(\sigma_{S D}^{2}, \sigma_{S T}^{2}\right.$ and $\left.\sigma_{S D T}^{2}\right)$. The sum of the three random error variances is called the relative error variance $\left(\sigma_{R E L}^{2}\right)$. Therefore, all the systematic error variances, $\sigma_{D}^{2}$ and $\sigma_{T}^{2}$, and the random error associated with their interaction $\sigma_{D T}^{2}$ are absent in the calculation. The generalizability coefficient for the D-study using the mean of five trials in one day is given by:

$$
\begin{aligned}
& \rho^{2}=\frac{\sigma_{\mathrm{S}}^{2}}{\sigma^{2}+\sigma_{\mathrm{REL}}^{2}} \quad \text { where } \\
& \sigma_{R E L}^{2}=\left(\sigma_{S D}^{2}\right)+\left(\sigma_{S T}^{2}\right) / 5+\left(\sigma_{S D T}^{2}\right) / 5
\end{aligned}
$$

When inter-subject variance $\sigma_{S}^{2}$ is high relative to the absolute or relative error variance, the reliability coefficients $\left(\rho^{2}\right.$ and $\left.\Phi\right)$ increase. The scores of these coefficients range between 0 and 1 , with 0 representing null reliability and 1 , perfect reliability. However, these coefficients can be high even when the measurement error is so large that, in some applications, the measurement cannot be used. This is why it is appropriate to report the errors in terms of the units of measure, which are given by the standard errors of measurement (SEM) [15]. Therefore this statistic was also computed for both types of reliability coefficients. The absolute standard error of measurement $\left(\mathrm{SEM}_{\mathrm{ABS}}\right)$ and relative standard error of measurement $\left(\mathrm{SEM}_{\mathrm{REL}}\right)$ are the root square of the absolute $\left(\sigma_{A B S}^{2}\right)$ and relative $\left(\sigma_{R E L}^{2}\right)$ error variances, respectively [15]. In the present study, the SEMs were described in N.m for the moment parameter, in degrees for the angle parameter and in $\mathrm{N} . \mathrm{m} /{ }^{\circ}$ for the stiffness coefficient. 
Table 2. Range of Percentages (\%) of Variance Calculated in the G-Study for the Moment (Second Column) and the Stiffness Coefficient (Fourth Column) at a Common Angle Across Subjects as Well as for the Angle (Third Column) at a Common Moment Across Subjects. The Common Angle or Moment was Specific to Each Knee Position. The Ranges Cover the Percentages Found at the Three Knee Positions

\begin{tabular}{|c|c|c|c|}
\hline & Moment & Angle & Stiffness Coefficien \\
\hline Sources of Variance & $\begin{array}{l}\% \text { of Variance } \\
\text { (Range) }\end{array}$ & $\begin{array}{l}\% \text { of Variance } \\
\text { (Range) }\end{array}$ & $\begin{array}{l}\% \text { of Variance } \\
\text { (Range) }\end{array}$ \\
\hline Subject $\left(\sigma_{s}^{2}\right)$ & $63.43-70.18$ & $61.03-77.14$ & $55.91-68.66$ \\
\hline $\operatorname{Day}\left(\sigma_{D}^{2}\right)$ & $6.50-8.55$ & $4.07-11.11$ & $0.10-9.50$ \\
\hline Trial $\left(\sigma_{T}^{2}\right)$ & $0.00-0.00$ & $0.00-0.14$ & $0.00-0.00$ \\
\hline Subject-day $\left(\sigma_{S D}^{2}\right)$ & $19.42-29.64$ & $15.31-25.63$ & $23.72-30.79$ \\
\hline Subject-trial $\left(\sigma_{S T}^{2}\right)$ & $0.00-0.07$ & $0.00-0.72$ & $0.00-0.77$ \\
\hline Day-trial $\left(\sigma_{D T}^{2}\right)$ & $0.00-0.00$ & $0.00-0.00$ & $0.00-0.23$ \\
\hline Subject-day-trial $\left(\sigma_{S D T}^{2}\right)$ & $0.35-1.81$ & $2.19-4.11$ & $1.22-4.84$ \\
\hline Total variance & 100 & 100 & 100 \\
\hline
\end{tabular}

The generalizability analysis was done with the GENOVA program (Crick and Brennan, 1983) ${ }^{1}$.

\section{RESULTS}

The G-study results (Table 2) revealed that the subject component represented the highest proportion of the total variance for the three stiffness parameters and this was observed for the three knee positions. The subject variable explained between $63.43 \%$ and $70.18 \%, 61.03 \%$ and $77.14 \%$ and, $55.91 \%$ and $68.66 \%$ of the total variance for the moment, angle and stiffness coefficient parameters, respectively. There was a notable random effect from the interaction between the subject and day facets, which represented the second most significant source of variance with values ranging from $15.31 \%$ to $30.79 \%$ depending on the stiffness parameter and the knee position. In addition, a systematic effect of the day facet was noted for the three stiffness parameters at the three knee positions. This systematic day effect, characterized by higher moments and stiffness coefficients as well as smaller extension angles or higher flexion angles on day 2, was the third most important source of variance and yielded $0.10 \%$ to $11.11 \%$ of the total variances. The lowest value $(0.10 \%)$ was obtained for the stiffness coefficient at the $\mathrm{K} 45^{\circ}$ knee position. The residuals (interaction between subject, day and trial facets) represented less than $4.84 \%$ of the total variances. However, for the $\mathrm{K} 45^{\circ}$ knee position, it was the third most important source of variance for the stiffness coefficient. Finally, Trial, Subject-Trial and Day-Trial facets represented less than $1.0 \%$ of the total variances.

The D-study (Table 3) for the mean of five trials measured in one day determined generalizability coefficients and dependability indices ranging from 0.58 to 0.83 . As expected, the generalizability coefficients were higher than the dependability indices. The values were in the same range for all parameters at the $\mathrm{K}^{\circ} 5^{\circ}$ and $\mathrm{K} 90^{\circ}$ knee positions while the

\footnotetext{
${ }^{1}$ Information can be obtained by writing to JE Crick, National Board of Medical Examiners. 3930 Chestnut St., Philadelphia, PA 19104. See also free software available at: www.uiowa.edu/ itp/pages/SWGENOVA. SHTML)
}

$\mathrm{K} 0^{\circ}$ knee position revealed the highest coefficients for the moment and angle parameters but the lowest for the stiffness coefficient. It should also be noted that the generalizability and dependability coefficients for the moment and angle parameters diminished slightly with the increase of flexion at the knee $\left(\mathrm{K} 0^{\circ}>\mathrm{K} 45^{\circ}>\mathrm{K} 90^{\circ}\right)$. The inverse was observed for the stiffness coefficients.

The highest SEM values for the three knee positions were $4.15 \mathrm{~N} . \mathrm{m}, 8.44^{\circ}$ and $0.23 \mathrm{~N} . \mathrm{m} /{ }^{\circ}$ for the moment, angle and stiffness coefficient, respectively. The SEMs for the moment were similar across the three knee positions, those for the angle parameter increased with the augmentation of the knee flexion, whereas those associated with the stiffness coefficients were highest at the $\mathrm{K} 0^{\circ}$ knee position. This is generally consistent with the variations in function of the knee position noted for the reliability coefficients.

\section{DISCUSSION}

The purpose of this study was to estimate the reliability of three passive stiffness parameters (moment, angle and stiffness coefficient) for the hip extension movement in healthy subjects for three different knee positions. The results indicated moderate to good coefficients $\left(\rho^{2}\right.$ or $\left.\Phi>0.5\right)$ according to the nominal scale suggested by Portney and Watkins [17] for intra-class correlation coefficients. The main error was related to both the systematic and random variations across days. For a particular random D-study design which consisted of five trials measured in one day, the SEMs were lower than $4.15 \mathrm{Nm}, 8.44^{\circ}$ and $0.23 \mathrm{~N} . \mathrm{m} /{ }^{\circ}$ for the moment, angle and stiffness coefficients, respectively.

Generally, the subject component represented the highest proportion $(55.91 \%$ to $77.14 \%)$ of the total variance for the three stiffness parameters and knee positions. These percentages are related directly to the reliability values because they are the proportion of the total variance free of measurement error. Thus, a high percentage of subject variance necessarily implies a low percentage of error variances and a high reliability coefficient. 
Table 3. Reliability Coefficients and SEMs for the Moment (Third Column) and the Stiffness Coefficient (Fifth Column) at a Common Angle Across Subjects as Well as for the Angle (Fourth Column) at a Common Moment Across Subjects. The Common Angle or Moment was Specific to Each Knee Position. The Values are for a D-Study Design Involving the Mean of Five Trials Measured in One Day

\begin{tabular}{|c|c|c|c|c|}
\hline Reliability Coefficients and SEMs & Knee Positions & Moment & Angle & Stiffness Coefficient \\
\hline Generalizability Coefficients $\left(\rho^{2}\right)$ & \multirow{4}{*}{$\mathrm{K} 0^{\circ}$} & 0.78 & 0.83 & 0.64 \\
\hline Dependability Indices $(\Phi)$ & & 0.71 & 0.79 & 0.58 \\
\hline $\mathrm{SEM}_{\mathrm{REL}}$ & & 3.43 & 5.27 & 0.20 \\
\hline $\mathrm{SEM}_{\mathrm{ABS}}$ & & 4.10 & 5.91 & 0.23 \\
\hline Generalizability Coefficients $\left(\rho^{2}\right)$ & \multirow{4}{*}{$\mathrm{K} 45^{\circ}$} & 0.75 & 0.72 & 0.71 \\
\hline Dependability Indices $(\Phi)$ & & 0.70 & 0.68 & 0.71 \\
\hline $\mathrm{SEM}_{\mathrm{REL}}$ & & 3.56 & 6.94 & 0.16 \\
\hline $\mathrm{SEM}_{\mathrm{ABS}}$ & & 4.05 & 7.67 & 0.16 \\
\hline Generalizability Coefficients $\left(\rho^{2}\right)$ & \multirow{4}{*}{$\mathrm{K} 90^{\circ}$} & 0.68 & 0.70 & 0.73 \\
\hline Dependability Indices $(\Phi)$ & & 0.64 & 0.62 & 0.66 \\
\hline $\mathrm{SEM}_{\mathrm{REL}}$ & & 3.76 & 7.07 & 0.15 \\
\hline $\mathrm{SEM}_{\mathrm{ABS}}$ & & 4.15 & 8.44 & 0.17 \\
\hline
\end{tabular}

The results of the G-analysis revealed that the variances associated with the trial facet $\left(\sigma_{T}^{2}, \sigma_{D T}^{2}, \sigma_{S T}^{2}, \sigma_{S T D}^{2}\right)$ represented a small percentage of the total variance. This could be explained by the fact that the subjects remained in the same position with the same setting of the experimental apparatus between each trial. A consequence of the low variance percentage associated with the trial factor is the negligible effect of the number of trials on reliability. Thus, a D-study design with less than five trials in one day should not change the reliability too much. In fact, computation of the reliability coefficients for a D-study using a mean of three trials gave exactly the same coefficients and SEM as for five trials. Therefore, hip stiffness could suitably be evaluated with three trials instead of five.

A notable random effect from the interaction between the subject and day facet $\left(\sigma_{S D}^{2}\right)$ was also found $(15.31 \%$ to $30.79 \%)$. In fact, this interaction was generally the most large source of error variance for the three parameters and knee positions. This random effect indicates inconsistent changes across days among different subjects; in comparison to their results on the first day, some had higher stiffness values on the second day, while others had reduced or equal values.

A systematic effect of the day facet $(0.10 \%$ to $11.11 \%)$, clearly shown in Fig. (3), was also revealed for the three stiffness parameters at each knee position. However, for the stiffness coefficient, this systematic day effect was not as important as for the other two stiffness parameters. This systematic day effect cannot result from a training effect caused by the stretching on the first day because higher moment and stiffness as well as lesser hip extension angle were found on the second day. The inverse would have been expected from a training effect.

Both systematic and random effects associated with the day facet can be explained by three factors. The first is the misalignment of the Biodex dynamometer axis with the hip axis. This creates relative movement between the Biodex and lower extremity with the result that the angle recorded by the Biodex is not the same as the hip angle. The second factor is the difference in the reference (neutral position) that is used to align the moment-angle recorded on both days. For example, if the reference angle is, by mistake, more in flexion on the second day than on the first day, this will shift the moment-angle curve of the second day towards hip extension. In this case, the stiffness parameters measured for the same value (i.e. angle for the moment and stiffness coefficient parameters and moment for the angle parameter) will be different even if both curves are similar in reality. Readers could better understand this point using Fig. (2) and assuming that the curve of subject \#1 is analogous to the curve recorded on the first day and the curve of subject \#2 analogous to the curve obtained on the second day. The last factor in the difference is the stabilization of pelvis movements. Because it is impossible to completely stabilize the pelvis when moving the thigh, the angle recorded by the Biodex is influenced by both the hip and pelvis movements. Lack of pelvic stabilization one day relative to the other could decrease the recorded stiffness because movement occurs in a flexible low-back region instead of in a stiff hip joint.

In order to control the adverse effects of the previous factors, we have developed an alternative approach to measure passive stiffness at the hip [18]. In this approach, the lower extremity is suspended by a cable and free to move horizontally. Forces are provided by a hand-held transducer while lower extremity and pelvis positions are simultaneously recorded by an optical system. It is expected that the reliability of this system will be better than that obtained in the present study.

The results of the D-study demonstrated that the reliability of the three stiffness parameters was generally moderate to good for the three knee positions, the reliability of the 
(A)

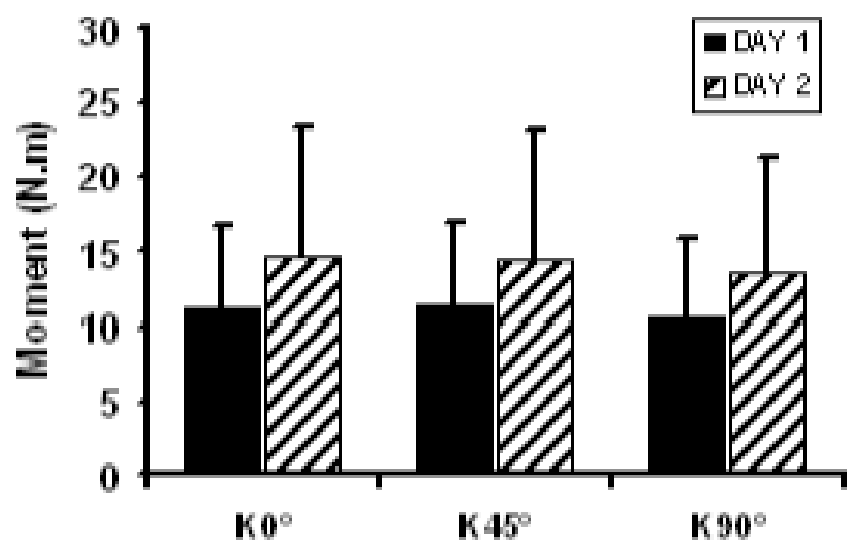

(B)

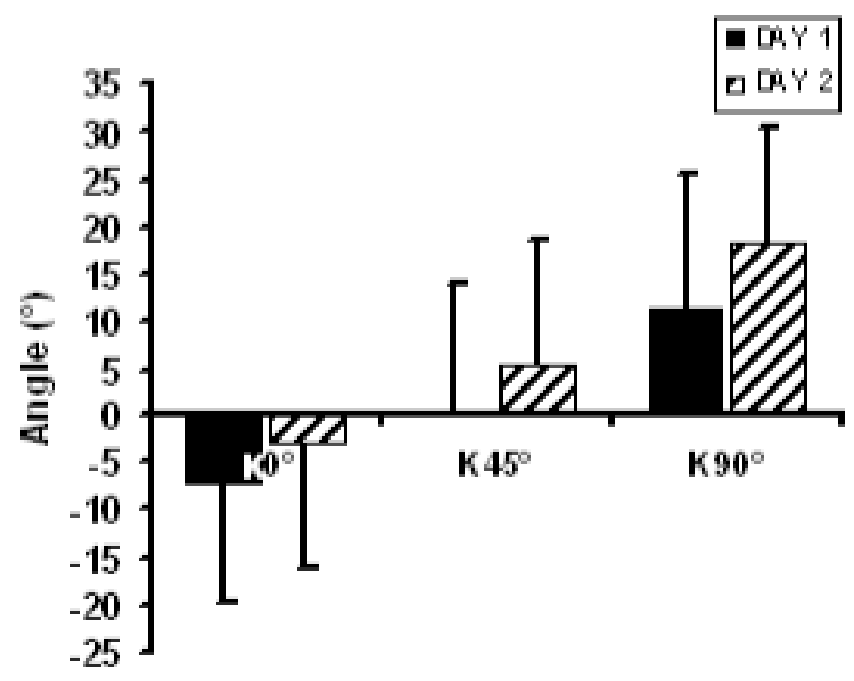

(C)

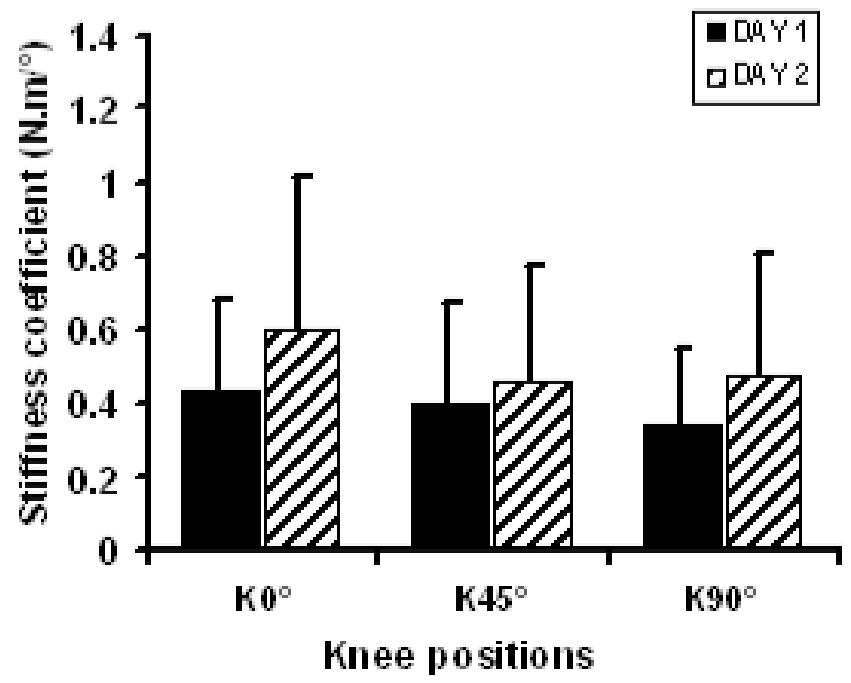

Fig. (3). Mean values and standard deviations of the moment (A), angle (B) and stiffness coefficient (C) parameters measured on day 1 and day 2 for the angles (moment and stiffness parameters graphics) and moments (angle parameter graphic) common across the subjects but specific to each knee position. For the angle parameter, negative values indicate hip extension angles. stiffness coefficient being slightly lower for the $\mathrm{K} 0^{\circ}$ knee position. This lower reliability of the stiffness coefficient for the $\mathrm{K} 0^{\circ}$ knee position could be explained by a lower subject variance for this condition $(55.91 \%)$ associated with an increase in the subject day variance $(30.79 \%)$.

With regards to the SEMs, the values reported for the moment coefficient ( 3.43 N.m to 4.15 N.m) are comparable to the average maximum variability test-retest reported in the study of Vrahas et al. [7]. For the other two parameters, no study has reported their associated SEM values. However, the $\mathrm{SEM}_{\mathrm{REL}}$ values obtained for the angle parameter can be compared with the errors associated with goniometric measurements. Boone et al. [19] found measurement errors of $3^{\circ}$ to $4^{\circ}$ for test-retest reliability, which is less than the corresponding $\mathrm{SEM}_{\mathrm{REL}}$ values found in this study $\left(5.27^{\circ}\right.$ to $\left.7.07^{\circ}\right)$. This is surprising considering that the moment applied to passively move the hip joint was carefully controlled, which is not the case in goniometric measurement. However, as explained above, the Biodex dynamometer used in this study to record the hip angles could not provide any information on the movement of the pelvis in relation to the femur, which is possible to control with goniometric measurements.

The small sample size $(n=20)$ of this study affects the accuracy of the estimation of the reliability coefficients. This statistic, like others, is subject to sampling fluctuations. At the present time, there is no procedure to calculate the confidence interval around the reliability coefficients for the twofactor design used in our study. Recent statistical developments in sample size estimation for designs involving one factor [20] could be used, in the future, as a basis for computing the confidence interval in complex designs. The values that are reported here are therefore an approximation of the instability of stiffness measurement.

\section{CONCLUSION}

Overall, the results of the G-analysis revealed that hip stiffness could be evaluated with three trials instead of five. An important random effect from the interaction between the subject and day facet was also found as well as a systematic effect of the day facet. Future studies will therefore need to better control the alignment of the hip rotation axis to reduce these errors on the stiffness parameters.

The results of the D-study demonstrated that the reliability of the three stiffness parameters was generally moderate to good for the three knee positions. These results suggest that these three stiffness parameters could therefore be used to evaluate hip stiffness. The measurement error found in the present study should also be taken into consideration, for example, to appreciate the real effects of stretching programs as well as the relationship between hip stiffness and other variables. Finally, the data on healthy subjects might provide reference values for comparison with patients with a hip pathology.

\section{ACKNOWLEDGEMENTS}

This research was completed at the Pathokinesiology laboratory of Dr. Gravel and Dr. Nadeau and was supported by a grant from the Canadian Institutes of Health Research (CIHR) and the Fonds de la recherche en santé du Québec (FRSQ). Anabèle Brière was supported by a M.Sc. Scholar- 
ship of the FRSQ and the Ordre Professionnel de la Physiothérapie du Québec (OPPQ). Dr. Nadeau had a Junior II investigator award of the FRSQ. The authors acknowledge Marie-Héléne Milot,.Daniel Marineau, Michel Goyette and France Piotte for their technical support.

\section{REFERENCES}

[1] Hof AL. Changes in muscles and tendons due to neural motor disorders: Implications for therapeutic intervention. Neural Plast 2001; 8: 71-81.

[2] Nadeau S, Gravel D, Olney SJ. Determinants, limiting factors, and compensatory strategies in gait. Crit Rev Phys Rehabil Med 2001; 13: $1-25$.

[3] Shimada T. Factors affecting appearance patterns of hip-flexion contractures and their effects on postural and gait abnormalities. Kobe J Med Sci 1996; 42: 271-90.

[4] Gleim GW, McHugh MP. Flexibility and its effects on sports injury and performance. Sports Med 1997; 5: 289-99.

[5] Johns RJ, Wright V. Relative importance of various tissues in joint stiffness. J Appl Physiol 1962; 17: 824-8.

[6] Gajdosik RL. Passive extensibility of skeletal muscle: review of the literature with clinical implications. Clin Biomech 2001; 16: 87101.

[7] Vrahas M, Brand R, Brown T, Andrews J. Contribution of passive tissues to the intersegmental moments at the hip. J Biomech 1990; 23: 357-62.

[8] Yoon YS, Mansour JM. The passive elastic moment at the hip. J Biomech 1982; 15: 905-10.
[9] Riemann BL, Demont RG, Ryu K, Lephart SM. The effect of sex, joint angle, and the gastrocnemius muscle on passive ankle joint complex stiffness. J Athletic Training 2001; 36: 369-75.

[10] Lee LW, Kerrigan KD, Della CU. Dynamic implications of hip flexion contractures. Am J Phys Med Rehabil 1997; 76: 502-8.

[11] Murray M, Gore D, Clarkson B. Walking patterns of patients with unilateral hip pain due to osteo-arthritis and avascular necrosis. J Bone Joint Surg 1971; 53: 259-74.

[12] Riener R, Edrich T. Identification of passive elastic joint moments in the lower extremities. J Biomech 1999; 32: 539-44.

[13] Harlaar J, Becher JG, Snijders CJ, Lankhorst GJ. Passive stiffness characteristics of ankle plantar flexors in hemiplegia. Clin Biomech 2000; $15: 261-70$.

[14] Roberson L, Giurintano DJ. Objective measures of joint stiffness. J Hand Ther 1995; 8: 163-6.

[15] Shavelson RJ, Webb NM. Generalizability theory: a primer Newbury Park, California: Sage Publications; 1991; vol. 1.

[16] Brennan R, Kane M. An index of dependability for mastery tests. J Educ Meas 1977; 14: 277-89.

[17] Portney LG, Watkins MP. Foundations of clinical research; Applications to practice. $2^{\text {nd }}$ ed. Upper Saddle River: Julie Alexander; 2000.

[18] Gaudreault N, Gravel D, Nadeau S, Desjardins P, Brière A. A method to evaluate contractures effects during the gait of children with Duchenne dystrophy. Clin Orthop Relat Res 2006; 456: 51-7.

[19] Boone DC, Azen SP, Lin CM, Spence C, Baron C, Lee L. Reliability of goniometric measurements. Phys Ther 1978; 58: 1355-90.

[20] Shoukri MM, Asyali MH, Donner A. Sample size requirements for the design of reliability study: review and new results. Stat Meth Med Res 2004; 13: 251-71. 